\title{
Performance Tuning of Coordinated Active Traffic Control Algorithm: Simultaneously Improving Corridor Safety and Mobility Performances
}

\author{
Jie Fang ${ }^{1,2}$ \\ ${ }^{1}$ College of Civil Engineering, Fuzhou University, Fuzhou, Fujian 350108, China \\ ${ }^{2}$ Department of Civil and Environmental Engineering, University of AB, 3-019 NREF, Edmonton, Alberta, Canada T6G 2 W2 \\ Correspondence should be addressed to Jie Fang; jfang1@ualberta.ca
}

Received 18 March 2014; Revised 15 June 2014; Accepted 16 June 2014; Published 9 July 2014

Academic Editor: Erik Cuevas

Copyright ( 2014 Jie Fang. This is an open access article distributed under the Creative Commons Attribution License, which permits unrestricted use, distribution, and reproduction in any medium, provided the original work is properly cited.

Proactive traffic control based on macroscopic traffic flow model is an innovative approach to active traffic management. An online, model predictive control (MPC) based active traffic control algorithm, DynaTAM, is proposed to implement integrated control through ramp metering (RM) and variable speed limit (VSL). DynaTAM predicts traffic states to anticipate incoming traffic congestion and to provide control plan recommendations for optimizing the network traffic conditions. However, as with other sophisticated prediction-based control algorithms, a system fine-tuning procedure is required for DynaTAM. In this study, two aspects will be addressed to further improve system performance. First, the control algorithm is evaluated to find the correlations between the prediction horizon length and the controlled system performance to suggest the most efficient prediction horizon length for the control algorithm. Second, safety considerations are quantitatively incorporated into the control algorithm. The control algorithm optimizes the traffic network targeting the cost reductions achieved by both improved mobility and reduced crash risk. A field-data-based simulation study is conducted to evaluate the system performance within various parameters and to determine the most suitable algorithm parameters. Optimized by the refined DynaTAM algorithm, the targeted area shows significant improvements in terms of both traffic safety and mobility.

\section{Introduction}

According to recent studies, traffic congestion is still one of the most challenging problems travelers and transportation agencies face. Daily recurrent congestion on freeways leads to significant mobility losses, as well as increased energy consumption and emissions. The Texas Transportation Institute (TTI) reports that, in 2010, the traffic congestion problem in America's metropolitan areas led to an additional 4.8 billion hours of travel time, consuming 1.9 billion gallons of extra fuel [1]. The total cost of congestion was over 100 billion US dollars. Another costly problem in transportation is crashes. Research from the American Automobile Association [2] reports that traffic crashes killed more than 33,000 people in the United States alone in 2010-that is about 93 deaths per day and nearly four every hour. Moreover, in 2009, these crashes cost about $\$ 300$ billion US dollars. In Canada, Transport Canada reports [3] about 300 fatalities and 200,000 injuries resulted from road collisions in 2007. In the studied area (Alberta, Canada), a total of 1,58,055 collisions were recorded during 2008 and the estimated fatal collisions were $0.2 \%$ (375) of total collisions reported by Alberta Transportation [4].

To mitigate traffic congestion and congestion-related crashes, the most important development in recent transportation management research is the concept of active traffic management (ATM). One major approach to implement ATM is through a traffic prediction model-based proactive traffic control system. In 2011, the City of Edmonton initiated a study to examine the feasibility and performance of implementing integrated active traffic management in Edmonton, Alberta, Canada. The work targets improving the traffic 
operations for a major highway corridor in Edmonton, which suffers from daily recurrent traffic congestion. The command framework, DynaTAM (Dynamic Analysis Tool for Active Traffic Demand Management), is aimed at performing prediction-based optimal control using both ramp metering (RM) and variable speed limit (VSL) techniques. Relying on an embedded macroscopic traffic state prediction model, DynaTAM predicts traffic states to anticipate incoming traffic congestion and provides control plan recommendations to optimize the network-wide traffic condition. The DynaTAM system has achieved significant improvements in terms of enhancing the mobility of the studied highway corridor [5]. As is the case with other sophisticated prediction-based control algorithms, however, DynaTAM needs to be carefully calibrated before it can be used in field implementation and achieve optimal system performance. This research presents the procedure of fine-tuning the DynaTAM control algorithm, mainly focusing on two tasks. The first task involves investigating the relationship between the length of the system prediction horizon (i.e., the future period of time in which the system performs traffic state predictions) and the system performance itself. This task depends on the analysis of system accuracy and computing complexity, determining the most efficient prediction horizon length for the control algorithm. The second task involves quantitatively considering the extent to which safety can be included in the control algorithm. The control algorithm will optimize the traffic network by deriving cost reductions from both the anticipated improved mobility and the reduced crash risk.

The rest of this paper is organized as follows: a literature review is conducted on the previous efforts of implementing active traffic control algorithm; the DynaTAM control algorithm and proposed tuning method are then presented; and finally, a field-data-based simulation study is included to demonstrate the tuning procedures of the DynaTAM control algorithm and to evaluate the optimized system performance.

\section{Literature Review}

Traffic engineers have long been dedicated to improving the efficiency of traffic operations using various traffic control measures. Among the resulting research, RM and VSL control techniques are the most widely studied and implemented in the field. RM control has been intensively studied in both Europe and the US. The earliest RM control algorithms include the demand-capacity strategy [6] and ALINEA developed by Papageorgiou et al. [7]. Both of these control strategies determine the traffic control plans according to the current traffic conditions. On the other hand, the VSL control measure has drawn much attention in recent years. Cassidy et al. [8] conducted a study to deploy the ramp metering at the upstream of the bottleneck location. Papageorgiou et al. [9] applied RM to facilitate real-time toll plaza and work zone management for achieving maximal throughput as well as to minimize delay. More recent RM studies, such as Kotsialos et al. developed in $[10,11]$, have extended the scope of control into the highway corridor. Another coordinated RM control algorithm, named HERO, was developed by Papamichail and Papageorgiou [12] and Papageorgiou et al. [13]. A three-layer hierarchical control structure was proposed to implement regional-coordinated RM by Papamichail et al. [14] .

In terms of safety improvements, early work on the VSL includes Zackor [15], which found that VSL control can reduce the speed difference between individual vehicles. Hellinga and Mandelzys [16] proposed a VSL control to prevent or postpone potential traffic breakdowns by taking advantage of the speed homogenization effect. Wang et al. [17] adopted an empirical approach to investigate the contribution of VSL control to improve operational safety. It was observed that VSL can improve the mobility of the highway during peak hours by enabling traffic flow with a higher density prior to traffic breakdown [16]. Another approach is to quantitatively examine the safety benefit through a statistical incident prediction model. Abdel-Aty et al. [18] developed a crash probability model which formulates the incident probability according to the traffic state measurements prior to the collision. The proposed crash probability model was subsequently adopted in $[19,20]$ to evaluate the performance of VSL control. Hellinga and Mandelzys [21] designed a control algorithm using decision tree with a similar collision model to implement VSL control. However, for most of the above mentioned studies, the applied controls were implemented targeting solely at either the mobility or the safety aspect of improvements. Fang et al. [22] proposed to use a prediction based VSL control to optimize the safety and mobility performance of a freeway corridor at the same stage.

As one solution to the growing traffic demand, transportation researchers have developed integrated control algorithms to implement multicontrol measures in their research. In terms of integrated control, early studies include Alessandri et al. [23], which predicted future traffic conditions using the Kalman filter. Abdel-Aty et al. [20] conducted research to implement integrated RM and VSL control, aiming at improving freeway mobility and reducing crash possibility during the congested period.

One major approach for implementing integrated RM and VSL is to perform a system-wide traffic optimization based on the future traffic status, which can be provided by a traffic flow prediction model. A famous early example is the implementation of the METANET model [24]. The authors used a macroscopic modeling approach to estimate the future traffic status. Hegyi et al. [16] conducted a study using a modified METANET to integrate RM and VSL. The original METANET model was modified to reflect the impact of the control measures on traffic. Therefore, the modified model could be used to perform traffic state prediction in control scenarios. Based on the predictions, the system can be optimized by simultaneously targeting total travel time (TTT) and total travel distance (TTD). Papamichail and Papageorgiou [12] combined RM and VSL for a large-scale highway network. The feasibility of the control algorithm was evaluated in a simulation study.

Recent efforts to integrate RM and VSL include Carlson et al. [25]. An algorithm named MTFC (mainstream traffic flow control) is proposed in this work, which combines VSL and RM using an extended METANET model. The targeted traffic system is optimized using an objective function consisting of TTT, TTD, and ramp penalty terms. Lu et al. [26] proposed 
another approach to combine VSL and RM design to improve the traffic operation at highway bottlenecks. In the control design, the traffic was optimized by minimizing the system mobility cost, while the safety performance of the system has not been the major focus of their work.

In summary, previous research shows that the efficiency of traffic operations can be improved by coordinately implementing RM and VSL. Nevertheless, the performance of a control algorithm is closely related to the quality of the system tuning. There are several essential components that require careful tuning to ensure the best performance of a proactive traffic control algorithm. First, proactive traffic control algorithm should be implemented based on the prediction results from macroscopic traffic flow model. The traffic flow model should be adopted by the control algorithm to perform predictions regarding the future traffic status over a certain period of time (the prediction horizon). With accurate prediction results, the system would be aware of congestion sooner, and necessary control decisions could be made before congestion occurs. Some previous researches suggest that the increased scope of the prediction horizon should lead to improved system performance with a considerable trade-off in terms of increased computing complexity. Traffic flow, however, is a sophisticated, dynamic system that undergoes rapid changes in status, meaning that the traffic conditions may change tremendously and not be at all related to the earlier traffic conditions. Without further experimentation, it is arbitrary to assume that the control algorithm performance will improve with the increased prediction horizon length. Consequently, in this inquiry, the underlying relationship between the length of the prediction horizon and the controlled system performance was examined. System performances possessing different prediction horizon lengths were evaluated and compared with the uncontrolled scenario benchmark.

Second, most previous studies focused on examining the control algorithm's performance in terms of the traffic mobility improvements; that is, the optimization criteria of the control algorithm (the defined objective function) usually aimed only at either minimizing the system-wide delay or maximizing the travel distance. Although some algorithms applied certain constraints to regulate the control plan with operational considerations [19-21], the safety impact of implementing the control measures has not yet been quantitatively considered, especially in terms of the control algorithm's optimization procedures. Therefore, to further optimize the performance of DynaTAM, a new form of the objective function is proposed to include safety costs in addition to the traditional system mobility indicators. This modification was made to achieve a better balance between the system mobility and safety improvements.

\section{DynaTAM Control Algorithm}

To provide the necessary background, the basic structure of the DynaTAM control algorithm is introduced in this section. For a detailed system design, please refer to Fang et al. [5, 22].

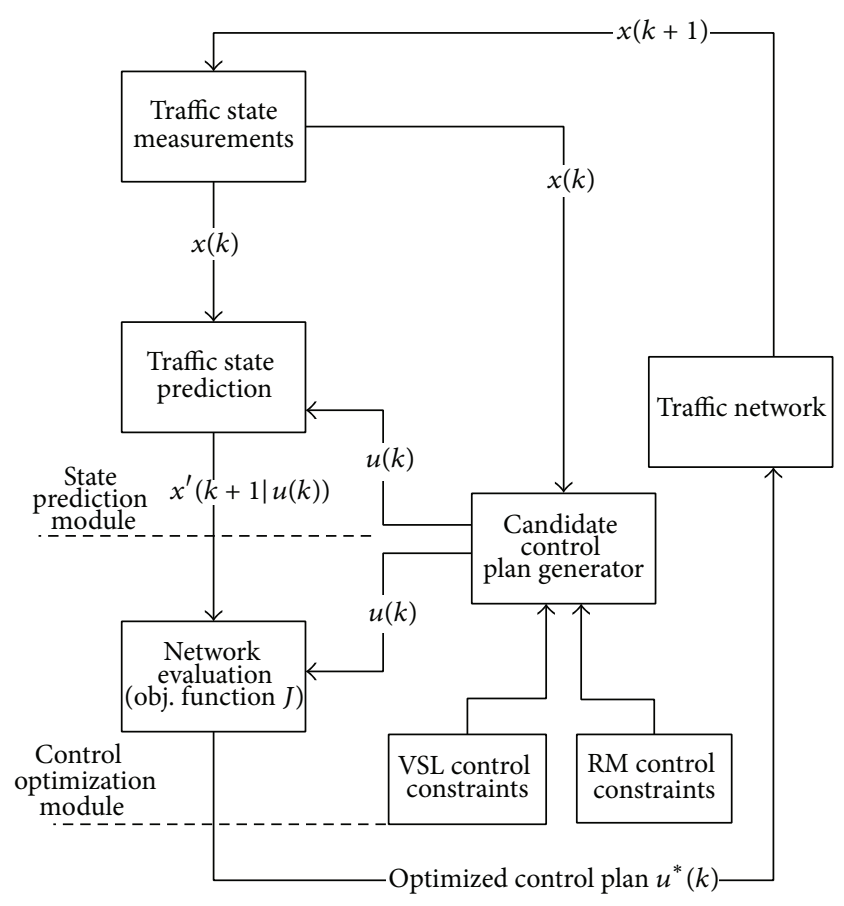

FIGURE 1: Framework of the DynaTAM control algorithm.

Figure 1 illustrates the basic system structures of the DynaTAM control algorithm. In brief, the DynaTAM control algorithm consists of key components.

Traffic State Prediction Module. This module serves as a macroscopic traffic flow model adopted to perform traffic condition predictions. The candidate control plan generator provides feasible control plan sets (regulated by certain field operational constraints) to the prediction module for analysis. The model takes the current traffic status $x(k)$ and the candidate control plan $u(k)$ as inputs to estimate the vector of the predicted future traffic condition, denoted by $x^{*}(k \mid u(k))$. The selected macroscopic traffic flow model divides the freeway into $m$ district links for analysis in $k$ continuous time steps. The adopted traffic state prediction model is inspired by the model in Lu et al. [26], which is a simplified version of the macroscopic traffic flow model, METANET:

$$
\begin{aligned}
\rho_{m}(k+1)= & \rho_{m}(k) \\
& +\frac{T}{L_{m} \lambda_{m}}\left[q_{m-1}(k)-q_{m}(k)+q_{\mathrm{on}}-q_{\mathrm{off}}\right], \\
q_{\mathrm{on}}= & \max \left(D_{\mathrm{on}, m}(k), u_{r, m}(k)\right), \\
v_{m}(k+1)= & v_{m}(k)+\frac{T}{\tau}\left(u_{v, m}(k)-v_{m}(k)\right) \\
& +\frac{T}{L_{m}} v_{m}(k)\left(v_{m-1}(k)-v_{m}(k)\right) \\
& -\frac{\mu T}{\tau L_{m}}\left(\frac{\rho_{m+1}(k)-\rho_{m}(k)}{\rho_{m}(k)+\kappa}\right),
\end{aligned}
$$


where $v_{m}(k), q_{m}(k)$, and $\rho_{m}(k)$ are the current link speed, flow rate and density, and critical density of link $m$, respectively:

$D_{\text {on, } m}(k)$ denotes the demand of on-ramp $m$ at time step $k$;

$T$ is the length of the time step, and $L_{m}$ and $\lambda_{m}$ are the length and lane number of link $m$;

$q_{\text {on }}$ and $q_{\text {off }}$ are the flow rate at the on-/off-ramp;

$u_{r, m}(k)$ and $u_{v, m}(k)$ are the system-permitted ramp flow rate and VSL value at link $m$;

$\tau, \mu$, and $\kappa$ are the model parameters.

Equation (1) is the flow conservation equation with the RM control term. Equation (2) estimates the incoming ramp flow and $D_{\text {on, } m}(k)$ is a random function added to the original model to predict the ramp demand based on the historical demand profile. Equation (3) is the speed prediction model that incorporates the VSL control; the link speed for the next time step $k+1$ was predicted in consideration of the advised VSL value and the traffic states in the adjacent links. For the details of model formulating, parameter calibrations and such, the author would refer to Lu et al's paper [26].

Control Optimization Module. This module determines the optimized control plan $u^{*}(k)$ based on the evaluation result of the system's performances. For each feasible control plan, the system performance is evaluated over the predefined system prediction horizon while subjected to the following constraints:

$$
\begin{gathered}
30 \mathrm{~km} / \mathrm{h} \leq u_{v, m}(k) \leq 80 \mathrm{~km} / \mathrm{h}, \\
\left|u_{v, m}(k)-u_{v, m}(k-1)\right| \leq 10 \mathrm{~km} / \mathrm{h}, \\
\left|u_{v, m}(k)-u_{v, m-1}(k)\right| \leq 20 \mathrm{~km} / \mathrm{h}, \\
0 \leq u_{r, m}(k) \leq Q_{\text {cap,ramp }}, \\
q_{m}(k)+u_{r, m}(k) \leq Q_{\text {cap }, m}, \\
u_{r}(k) \geq u_{r, \text { min }} \quad\left(\text { when } w_{o}(k)>w_{\text {cap }} * 95 \%\right), \\
u_{r, m}(k) \in(200,400,600, \ldots, 1200 v \mathrm{ph}), \\
u_{v, m}(k) \in(30,40,50, \ldots, 80 \mathrm{~km} / \mathrm{h}),
\end{gathered}
$$

where $u_{r, m}(k)$ and $u_{v, m}(k)$ are the system-permitted ramp flow rate and the VSL value on link $m$, respectively, both at time step $k$; $Q_{\text {cap,ramp }}$ and $Q_{\text {cap }, m}$ are the capacity of the onramp and freeway link $m ; q_{m}(k)$ is the flow rate of link $m$ at time step $k ; u_{r \text {,min }}$ is the predefined minimal ramp metering rate; and $w_{o}(k)$ and $w_{\text {cap }}$ are the queue length and the storage capacity at the on-ramp.

Constraints (4) to (6) were designed to regulate VSL control, which defines the boundary and variance for the VSL control signal. These constraints are designed to avoid the potential safety risks caused by abruptly changing the advised VSL. Constraints (7) to (9) regulate RM control by defining the boundary of the applicable ramp flow rate so it
(1) will not exceed the ramp capacity and (2) considers the limited storage space for each ramp. Constraints (10) and (11) further narrow the selection of the control plan into a series of predefined rounded numbers. This is designed to avoid the abrupt change of control signal and drivers' confusion caused by an unregulated speed limit number.

A system-defined objective function $J\left(x^{*}(k) \mid u(k)\right)$ is designed to reflect the optimization emphasis of the system operator. The system's performances (evaluated for each time step of the prediction horizon and accumulated for each control plan) are then compared to determine the most suitable control plan. At the next time step, the entire prediction-evaluation-optimization procedure will start all over again with the updated system status measurements.

In summary, the DynaTAM control algorithm determines the control plan according to its corresponding traffic network performance within the prediction horizon as it is measured by the objective function. Therefore, to tune the control algorithm to perform better, two critical questions must be raised.

(1) What is the relationship between the length of the prediction horizon and the controlled traffic system performance and, by implication, what is the most efficient prediction horizon selection method (accounting for both the system performance and the computational cost)?

(2) How can the objective function be designed for the evaluation model, that is, how can system mobility and safety gains be quantitatively analyzed?

In the following section, these two questions are investigated separately.

\section{Control Algorithm Tuning}

4.1. Conditions of the Benchmark Case. To evaluate the system performance during the tuning procedures, a field-databased simulation was conducted as a benchmark comparison scenario. The location of the benchmark simulation is Whitemud Drive in Edmonton, Alberta, Canada. As shown in Figure 2, Whitemud Drive is a main east-west freeway approximately 11 kilometres $(\mathrm{km})$ long in south Edmonton.

Functioning as part of the City of Edmonton's inner ring road, Whitemud Drive suffers from daily recurrent traffic congestion during peak time of days. In the target area, there are four on-ramps that can be managed by RM. As designated in Figure 2, two bottlenecks were identified along the corridor and the VSL control was carried out before and after the bottlenecks, respectively. Traffic data were collected using loop detectors along the corridor at 20-second intervals. Whitemud Drive was reproduced in VISSIM, a traffic simulation software, for control implementation and evaluation. With necessary calibration, a complete peak-hour demand profile (7:00-9:30 AM, see Figure 3) was configured in VISSIM to reproduce the real-world congested traffic environment. The DynaTAM control algorithm was implemented in this benchmark case to perform an integrated control of four RM and VSL changes every 60 seconds. It 


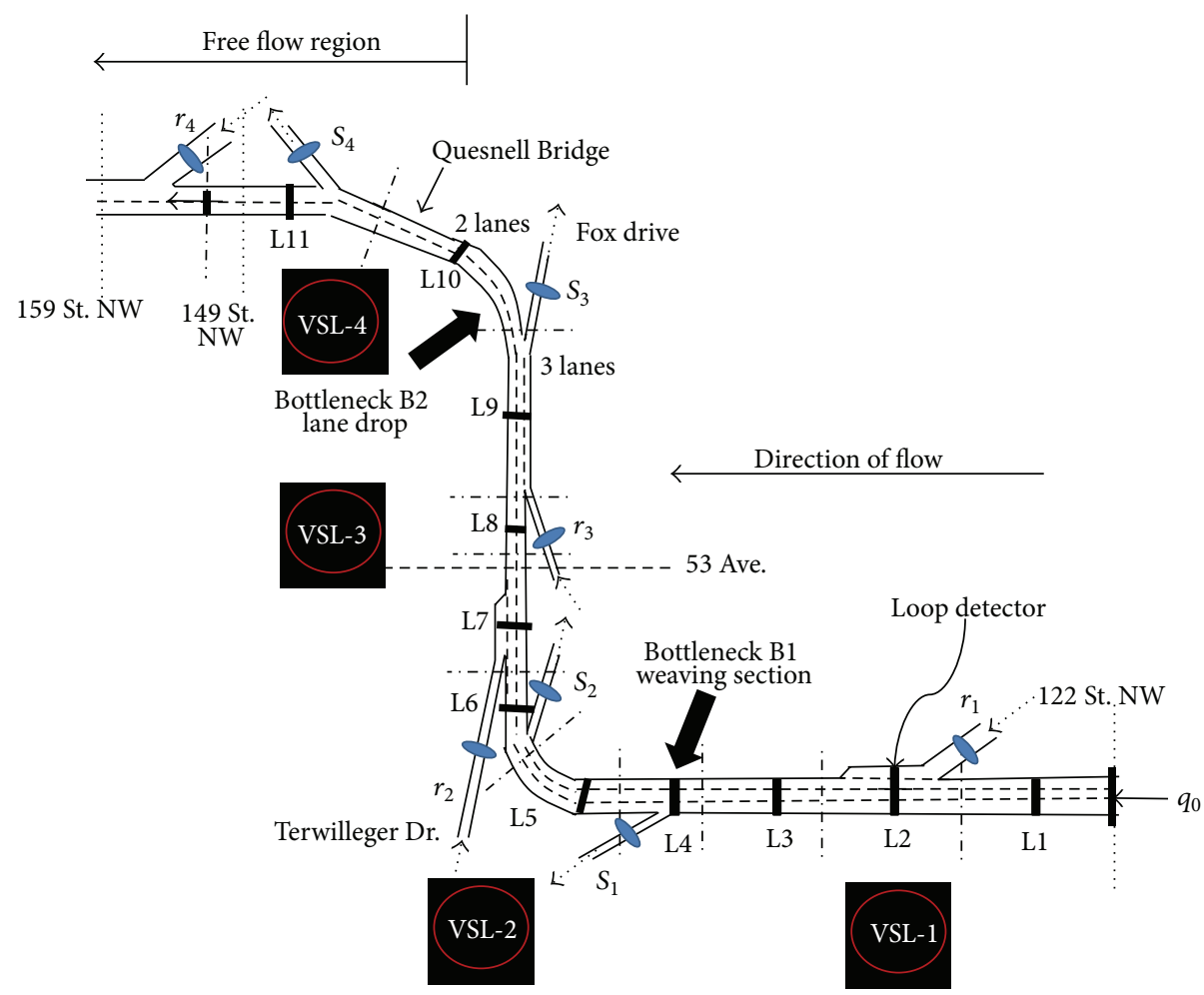

Mainline loop detector location

Ramp loop detector location

FIGURE 2: Site of application study: Whitemud Drive.

should be mentioned that this interval is connected with another important parameter in the MPC control design: the control horizon. Since the control signal should remain unchanged during the control horizon, the control horizon is directly associated with the frequency of changing the control signal (the advised variable speed limit value, more specifically). That is to say, unlike the prediction horizon, the configuration of the control horizon is strictly limited by the drivers comprehension and reaction mechanism. As a study targeting at field practices, the change of advisory variable speed limit value should not be too frequent for the driver to adapt. Thus, in the proposed algorithm, the advised speed limited was restricted to change at an interval of 1 minute, which is the length of control horizon as well.

4.2. Tuning the Prediction Horizon Length. To analyze the relationship between the prediction horizon length and the system performance and computational cost, the control algorithm was configured to run with a prediction horizon length that increased from 5 to 30 minutes. The system performances of these different scenarios were compared with the benchmark case using two measurements: (1) time cost to perform the system optimization and (2) system mobility level.

The optimization time was measured by recording the time consumed, while the system was generating the optimized control plan in each control interval $(60 \mathrm{sec}$.). The

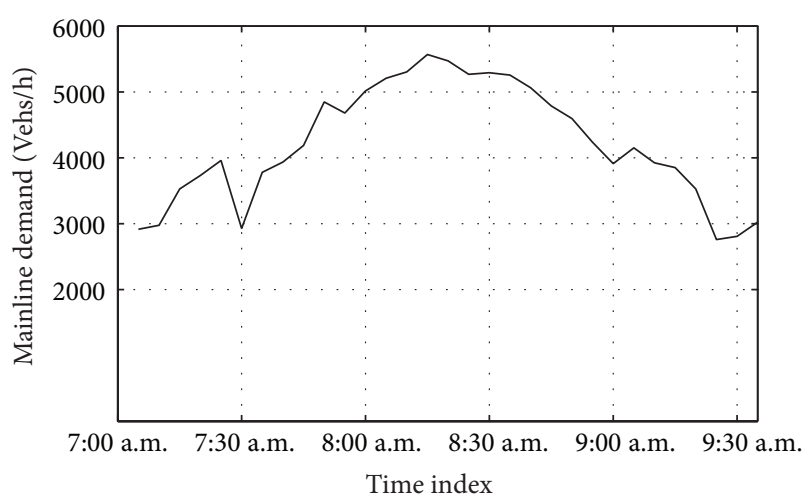

FIGURE 3: Demand profile of the experimental study.

simulation was run on a regular office desktop with Intel Core 7, 2.8 GHz, $8 \mathrm{~GB}$ RAM, and 64-bit Windows 7 system. The mobility cost item evaluates the system performances in terms of improvements in mobility. The mobility cost is measured by the following equation:

$$
\begin{gathered}
J\left(x^{*}(k) \mid u(k)\right)=\sum_{m} \sum_{k}\left(\alpha_{\mathrm{TTS}} \mathrm{TTS}-\alpha_{\mathrm{TTD}} \mathrm{TTD}\right), \\
\mathrm{TTS}=T * L_{m} * \lambda_{m} \sum_{m} \rho_{m}(k)+T * w_{0}(k), \\
\mathrm{TTD}=T * L_{m} * \lambda_{m} \sum_{m} \rho_{m}(k) v_{m}(k),
\end{gathered}
$$


TABLE 1: Comparison of system performances with different prediction horizon lengths.

\begin{tabular}{|c|c|c|c|c|c|c|c|}
\hline & $\begin{array}{l}\text { Benchmark case } \\
\text { (uncontrolled) }\end{array}$ & 5 minutes & 10 minutes & 15 minutes & 20 minutes & 25 minutes & 30 minutes \\
\hline Optimization time (sec.) & $\mathrm{N} / \mathrm{A}$ & 3.6 & 7.5 & 11.2 & 15.2 & 17.3 & 22.1 \\
\hline Mobility cost (objective function) & 911.4 & 466.6 & 390.1 & 517.7 & 2055.2 & 2160.9 & 2274.9 \\
\hline $\begin{array}{l}\text { Mobility improvements compared to } \\
\text { the benchmark case }(\%)\end{array}$ & N/A & 48.8 & 57.2 & 43.2 & -125.5 & -137.1 & -149.6 \\
\hline
\end{tabular}

where $\sum_{m} \sum_{k}$ is the set of all $m$ links and $k$ time steps in the studied network.

$$
\begin{aligned}
& \text { TTS and TTD are the total time spent and total travel } \\
& \text { distance; } \\
& \alpha_{\text {TTS }} \text { and } \alpha_{\text {TTD }} \text { are the empirical weighting factors for } \\
& \text { balancing TTS and TTD; } \\
& L_{m} \text { and } \lambda_{m} \text { are the length and lane number of link } m \text {; } \\
& T \text { is the length of the time step; } \\
& w_{0}(k) \text { is the length of the queue on the on-ramp. }
\end{aligned}
$$

Equation (12) measures the system's mobility cost, and it serves as the objective function for the system optimization procedure. The TTS and TTD are scaled to equivalent values using the weighting factor. Achieving the minimized value of this objective function indicates that the system obtained the optimal mobility cost by simultaneously minimizing the TTS and maximizing the TTD. The traffic system performance under DynaTAM control and with different prediction horizons (ranging from 5 to 30 minutes) was evaluated. The results are compared in Table 1.

As illustrated in the summarized table, all the scenarios are applicable online, since their reported optimization times are less than $50 \%$ of the control interval (60 seconds). Therefore, the length of the prediction horizon may be chosen according to the system performance. Furthermore, in scenarios with 5-, 10-, and 15-minute prediction horizons, the overall system performance was improved in terms of mobility. The reduced mobility cost (compared to the benchmark case) indicates that the DynaTAM system is functioning properly to regulate the traffic. However, in scenarios with prediction horizons longer than 20 minutes, the system performance is worse than the benchmark case. The reason for this is the inappropriately long prediction horizon, which causes the traffic prediction model to overestimate the variations in traffic states. As a result, the system is oversensitive to the changes in traffic measurements and implements unnecessarily strict regulations (e.g., low VSL value or RM rate) for the whole test duration, which limits system mobility. The detailed trend of system performance changes can be observed in Figure 4.

In Figure 4, it is observed that the scenarios with 5, 10-, and 15-minute prediction horizons have significantly better performances than the 30 -minute prediction scenarios. However, the scenario with 30-minute prediction horizons shows that the applied control has negative impacts on traffic efficiency. The reason is that for the 30-minute control scenario, the control algorithm tends to overestimate the

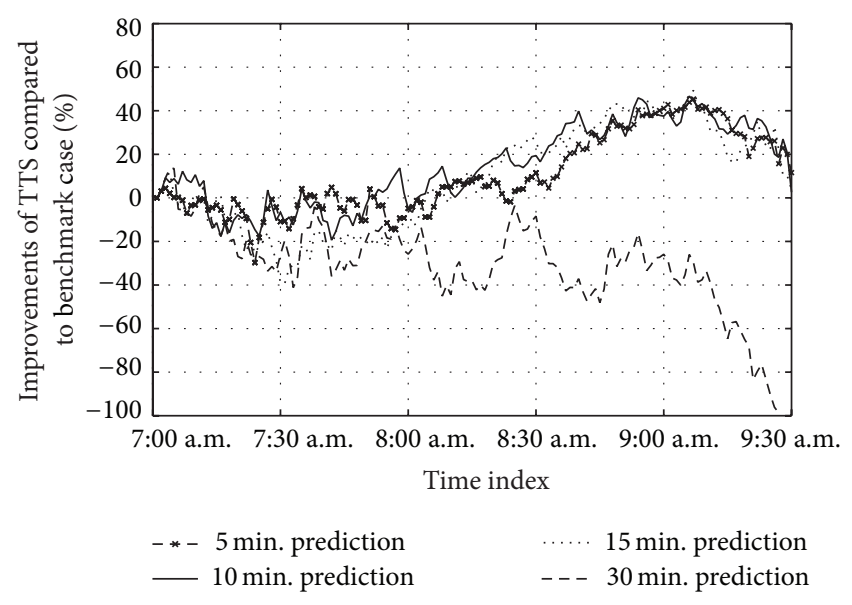

FIGURE 4: Improvements to mobility costs compared to the benchmark case (in \%).

severity of the traffic congestion. Although the change could be minor for a single time step, what makes it worse is the implemented low speed limit during not-so-congested period caused more and more demand lining up and the trend of overestimation accumulates very fast as the time moves on in the field. As a result, the advised speed limit was restricted at low values for most of the study duration. Thus, the demand cannot be efficiently discharged and the traffic flow was stuck in the congestion state.

For the three scenarios with improved traffic conditions, the DynaTAM control algorithm detected the incoming congestion and regulated it beforehand (between 7:15 and 7:45 AM, the time period is slightly different in each scenario due to a different prediction horizon), as was the intention of the control algorithm design. During this period, traffic regulation was implemented to restrict the traffic and prevent its breakdown. As a result of the implemented control measures (i.e., lowered speed limit and RM rate), the controlled system performance was worse than the benchmark case during this precongestion period. In return, the controlled scenarios were able to gain significant performance improvements of up to $50 \%$ later on during the congestion period (8:00-9:30 AM). Comparing the 5-, 10-, and 15-minute prediction scenarios, the trend of system performances is similar. Nevertheless, the system with 10-minute prediction performs better in terms of mobility than the 15-minute scenario for the precongestion period while gained as much in the congestion period. This observation also coincides with the results shown in Table 1, which indicate that a 10 -minute prediction case has the 
most significant performance improvements. Therefore, for DynaTAM in this specific study, 10 minutes was identified as the optimal prediction horizon length for the optimal mobility performance. Note that as the system performance may vary in scenarios with different demand levels and traffic flow characteristics, this optimal prediction horizon should be carefully evaluated before the implementation.

\subsection{Incorporating Mobility with Traffic Safety Considerations.} Another main objective of the system tuning in this study is to investigate how to evaluate system gains derived from both the mobility and safety aspects. Furthermore, this study aims to understand the interrelationship between traffic safety and mobility and how they can be incorporated. To investigate this problem, a new objective function is proposed in DynaTAM's optimization procedures to aim at both system mobility and safety. The proposed objective function $J$ reads

$$
J=\sum_{m} \sum_{k}\left(\text { Cost }_{\text {Mobility }}+\text { Cost }_{\text {Safety }}\right) \text {. }
$$

Equation (13) expresses that the control algorithm is designed to optimize the traffic network by minimizing two costs: the mobility cost Cost $_{\text {Mobility }}$ and the safety cost

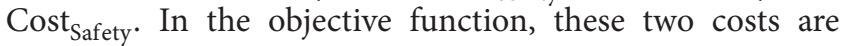
measured over the entire studied network $\left(\sum_{m}\right)$ and studied period $\left(\sum_{k}\right)$. The calculation of these two costs is shown in

$$
\begin{aligned}
& \text { Cost }_{\text {Mobility }} \\
& =R_{\text {Mobility }}\left(\alpha_{\text {TTS }} \text { TTS }-\alpha_{\text {TTD }} \text { TTD }\right) \\
& =R_{\text {Mobility }}\left\{\alpha_{\text {TTS }}\right. \\
& *\left[T * L_{m} * \lambda_{m} \sum_{m} \rho_{m}(k)+T * w_{0}(k)\right] \\
& \left.-\alpha_{\text {TTD }} *\left[T * L_{m} * \lambda_{m} \sum_{m} \rho_{m}(k) v_{m}(k)\right]\right\}, \\
& P_{\text {Incident }}=\frac{\operatorname{Cost}_{\text {Safety }}=R_{\text {Safety }} P_{\text {Incident }}}{1+\exp ^{\left(\text {Constant }-b_{1} S V_{2}+b_{2} \log A O_{1}+b_{3} \log S S_{2}\right)}},
\end{aligned}
$$

where $P_{\text {incident }}$ is a real-time model to estimate the incident probability.

$\mathrm{SV}_{2}$ is the standard deviation of volume in the last 5-10 minutes; $A O_{1}$ is the average occupancy in the previous $0-5$ minutes; and $S_{2}$ is the standard deviation of speed in the last 5-10 minutes. The calibrated values of the constants $b_{1}, b_{2}$, and $b_{3}$ are $-1.207,3.149,4.028$, and -3.694 , respectively.

In (14), the total system mobility cost was measured, converting TTD and TTS into equivalent values and computing the mobility cost (equivalent TTS) of the traffic network. Essentially, the tuning of these two parameters defines which element of traffic flow will the system favor. The system will tend to allow higher density if a larger weight of TTS ( $\alpha$ TTS) was assigned to the equation and otherwise a higher throughput to discharge the demand faster. Thus, there is no fixed optimum value for these two parameters, but the ratio of these two parameters is usually tuned to be the critical speed. Furthermore, these two elements of the traffic flow (density and outflow) are not entirely conflicting, so that the author chose to use the form of weighted summary, which means that a desired traffic state should favor both of the two MOEs at the same time. This approach of formulating the objective function is also common in the existing MPC based traffic control researches ([16, 25] and such). The safety cost term measures the total system safety cost by examining the system crash probability. A real-time incident probability model, proposed in [27], is adopted to assess traffic safety risks. The adopted model is a real-time crash probability assessment model developed and calibrated for the same Whitemud Drive study area. It used the matched case-control logistic regression technique to estimate crash likelihood. Crash likelihood was estimated based on the dynamics of traffic measurement (changes in volume, average occupancy, and standard deviation of speed). The traffic state variables 30 minutes prior to the incident occurrence were selected and divided into six 5 min time slices. These traffic state variables were indexed as time slices $1-6$. Index 1 is the 5 min slice right before collision occurrence.

To conduct the model, two years of collision data, which included 46 collisions at the experimental site, were used. The calibrated values of the constants $b_{1}, b_{2}$, and $b_{3}$ are $1.207,3.149,4.028$, and -3.694 , respectively. For more detailed descriptions of the model, please refer to the original paper.

$R_{\text {Mobility }}$ and $R_{\text {safety }}$ are two adjustable weighting factors introduced to measure and balance the cost of mobility and safety. The value of these two parameters determines the emphasis of the system optimization on mobility or safety. Table 2 shows the different value combinations for these two parameters as tested in this study. The logic behind selecting these numbers is found in prior empirical studies and incident data. For example, according to the 2011 TTI travel mobility report [1], the average cost of traveling one hour on the road is $\$ 8$. This number was selected as the baseline number to measure the value of TTS. The AAA crash report [2] reveals that the average cost for each incident in large metropolitan cities is around \$3800. Therefore, $\$ 3000$ was selected as the baseline averaged incident cost for measuring the safety performance of the network. In addition, the parameter setting was adjusted to evaluate the sensitivity of the parameter and to generate several scenarios for comparison (1000 and 5000) with the uncontrolled case and the scenario focusing on the extent of system mobility only $\left(R_{\text {safety }}=0\right.$, Scenario 2$)$. The selected scenarios were compared using both safety (crash probability) and mobility (TTS and TTD) measurements, as shown in Table 2.

The table indicates that, by implementing the DynaTAM control algorithm with the proposed objective function and safety considerations, the crash probability was lowered by $3-5 \%$. Given that the average crash probability for the uncontrolled scenario is approximately $11 \%$, crash risks were significantly reduced (by as much as $43 \%$ ). Moreover, this 
TABLE 2: Scenarios tested with different $R_{\text {mobility }}$ and $R_{\text {safety }}$ values.

\begin{tabular}{|c|c|c|c|c|c|}
\hline & Scenario 1 (uncontrolled) & Scenario $2^{*}$ & Scenario 3 & Scenario 4 & Scenario 5 \\
\hline$R_{\text {mobility }}$ & N/A & 1 & 8 & 8 & 8 \\
\hline$R_{\text {safety }}$ & N/A & N/A & 1000 & 3000 & 5000 \\
\hline Crash probability & $11.6 \%$ & $9.3 \%$ & $7.5 \%$ & $6.6 \%$ & $9.7 \%$ \\
\hline Mobility cost & 911.4 & 390.1 & 487.6 & 551.4 & 597.0 \\
\hline Mobility cost comparisons ${ }^{* *}$ & N/A & $-57.2 \%$ & $-46.5 \%$ & $-39.5 \%$ & $-34.5 \%$ \\
\hline
\end{tabular}

Note: ${ }^{*}$ in this scenario, the objective function considers only the mobility cost (i.e., $R_{\text {mobility }}=1$ and $R_{\text {safety }}=0$ ).

** Compared to the uncontrolled condition, mobility costs are measured by (14).

improved safety is achieved while ensuring that system mobility also improved. By evaluating the system mobility cost, it was found that the controlled system mobility performances improved more than 30\%. The most significant system mobility improvement was reported in Scenario 2, which did not consider optimizing the safety aspect of the system $\left(R_{\text {safety }}=0\right)$. This indicates that, to reduce the safety risks, some system mobility must be sacrificed by the control algorithm.

Another interesting discovery is that the crash probability did not appear to have a linear relationship with the increased safety cost weighting factor $\left(R_{\text {safety }}\right)$. It is observed that, with $R_{\text {safety }}$ lower than that of Scenario 5, Scenario 4 achieved the lower crash probability as well as better system mobility performances. In the adopted model, the crash probability is affected by three parameters: standard deviation of volume, standard deviation of speed, and the average occupancy. This indicates that traffic safety favors a system with stable and efficient traffic flow. Rapidly changing flow speed (higher standard deviation) and congestion (higher occupancy) will lead to a higher crash probability, which implies that the traffic safety level does not explicitly conflict with the increased traffic speed or volume (the traffic mobility level). A system with a higher mobility level does not necessarily lead to a decreased safety level. In fact, when the system is more congested, as it is in Scenarios 1 (no control) and 5 (control is too conservative due to the high $R_{\text {safety }}$ ), the crash probability was observed to be higher than in the other scenarios.

This finding indicates that, although tradeoffs exist, improving traffic mobility is not entirely at odds with improving traffic safety. For instance, during the congested period, the control algorithm will lower the speed limit at the bottleneck. Appropriately, lowering the speed limit enhances the speed homogenization effects, which leads to potentially higher capacity (improved mobility) as well as lower crash risks (improved safety). Thus, in some cases, the system could be optimized to target both mobility and safety through an appropriately tuned system objective function. Based on the above comparisons, Scenarios 3 and 4 were found to perform better overall in terms of both system safety and mobility performance. As a result, the parameters used in Scenario 4 (with the lowest crash risks and betterthan-average system mobility levels) were used for the rest of the study. Nevertheless, the road operators can select alternative parameter values according to an identified focus on providing either maximized mobility or safety.

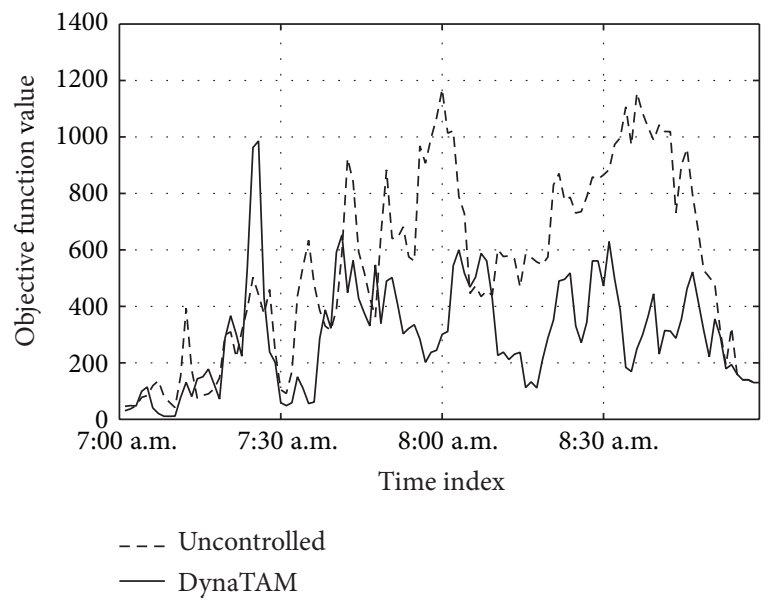

FIGURE 5: Objective function value comparisons between the uncontrolled and DynaTAM controlled scenarios.

\section{System Performance Evaluation}

After finalizing the system control and optimization parameters, the performance of the DynaTAM control algorithm was fine-tuned. This section presents the detailed outcome of this tuning process. Firstly, the overall system performance was examined through selected measurements and compared with the uncontrolled scenario. The results are shown in Table 3.

Table 3 shows that the refined DynaTAM control algorithm improved traffic conditions on the targeted highway corridor in terms of both mobility and safety. During the test period, the mobility and safety costs were reduced significantly by $39 \%$ and $43 \%$, respectively. When measuring the system performance (using the adopted objective function), it was shown that the DynaTAM-controlled scenario outperformed the uncontrolled case by more than $40 \%$. These improvements were achieved by both carefully tuning the prediction horizon length and simultaneously considering the traffic safety and mobility costs in the system optimization stage. Figure 5 visually demonstrates the improvement achieved over the course of the test period.

Figure 5 compares the system performances (measured by the proposed objective function) of the uncontrolled and DynaTAM-controlled scenarios over the entire test duration. The DynaTAM-controlled scenario significantly reduced the 
TABLE 3: Evaluation of optimized system performance.

\begin{tabular}{lccccc}
\hline & TTS (vehicle $*$ hr) & TTD $(\mathrm{km})$ & Mobility cost $^{1}$ & Crash probability $^{2}$ & Objective function $^{3}$ \\
\hline Uncontrolled & 3281.4 & 189598.4 & 911.4 & $11.6 \%$ & 7639.2 \\
DynaTAM & 2887.6 & 195855.1 & 551.4 & $6.6 \%$ & 4522.4 \\
Compared to uncontrolled case & $-12.0 \%$ & $+3.3 \%$ & $-39.5 \%$ & $-43.2 \%$ & $-40.8 \%$ \\
\hline
\end{tabular}

Note: ${ }^{1}$ measured by (14);

${ }^{2}$ relative improvements in $\%$;

${ }^{3}$ relative improvements in $\%$, measured by (13).

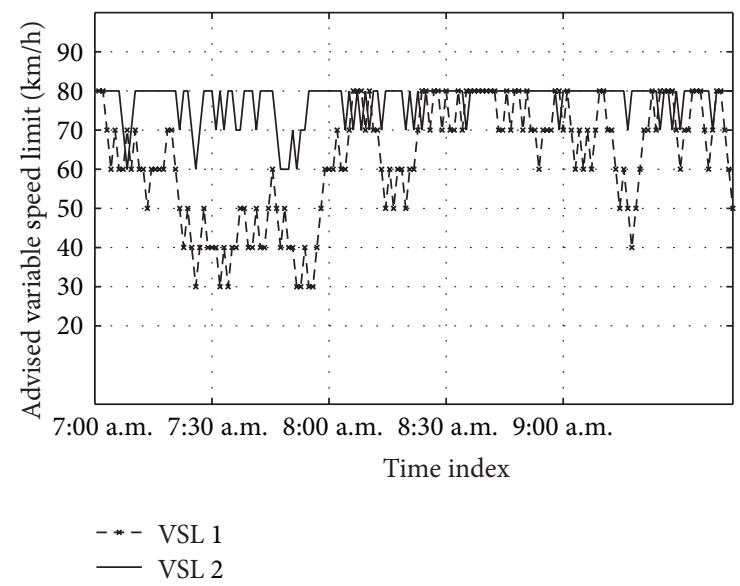

FIGURE 6: Advised variable speed limit value at VSL 1 and VSL 2.

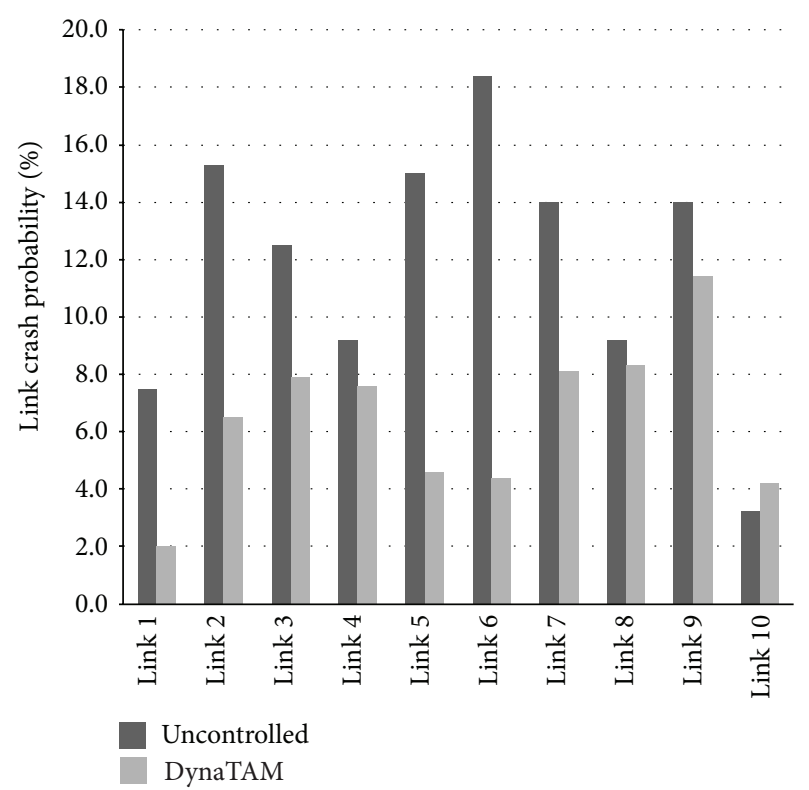

FIGURE 7: Link crash probability comparisons between uncontrolled and DynaTAM controlled scenario.

overall cost (considering both mobility and safety). During the uncongested period (before 8:00 AM), the system performance of the two scenarios was roughly the same, since there was no major control applied by DynaTAM. However, right before the congestion occurred (before 7:30 AM, see Figure 6 below), it is observed that in the controlled case the system cost increased before the uncontrolled case did.

It is observed that in the controlled case the system cost increased before the uncontrolled case did. This increase can be traced to DynaTAM detecting the incoming traffic breakdown and implementing control measures beforehand. The appropriate system prediction horizon allows sufficient time to optimize and deploy the countermeasure for the detected traffic breakdown. Based on the proposed new objective function, the RM and VSL control plans were selected to provide a combined optimal system for reducing safety and mobility costs over the entire prediction horizon. In Figure 6, it shows that the speed limit at the upstream of bottleneck location (VSL 1) was restricted during 7:00 to 8:00 while the downstream location still allows a higher speed limit $(60-70 \mathrm{~km} / \mathrm{h})$ during that period and throughout most of the experiment. Although the controlled system cost was higher for a short period before the congestion because of the proactive controls, it enabled more benefits in the congestions that followed. By restricting the traffic (i.e., lower posted speed limit or RM rate), the controlled network allowed more traffic throughout while lowering the crash risks (as the result of speed homogenization effects). These led to a noticeable overall system cost reduction during the congested period (8:00-9:30 AM in this study).

Since it is easy to understand that the system mobility cost will be lower when congestion is mitigated, Figure 7 is included to demonstrate the reduced system crash risks.

Figure 7 visually demonstrates that, in the DynaTAMcontrolled scenario, the crash probability for the tested corridor is efficiently reduced. Link 3 and link 8 are the locations of the two identified bottlenecks. It is observed that, in the uncontrolled case, the crash probability of these two links and their upstream links are noticeably high. Such high crash probabilities are caused by the abrupt traffic condition changes related to congestion in these links. In the controlled scenario, since DynaTAM adopted a new objective function to take the safety aspect of the optimization procedure into consideration, the traffic was restricted by the system upstream of the bottleneck locations. The lowered speed limit and $\mathrm{RM}$ rate sufficiently mitigated the traffic congestion to stabilize the traffic flow, which led to the observed reduction in crash probability.

\section{Conclusions}

This study presents the fine-tuning procedures of an integrated active traffic control algorithm, DynaTAM. The study 
focused on tuning the system in two ways: (1) by discovering the relationship between the system prediction horizon and the controlled performance to determine the suitable prediction horizon length; (2) by investigating both the traffic safety and mobility costs and taking both into consideration when optimizing the traffic network. A simulation was conducted as the benchmark case to evaluate performances in different tested scenarios.

To investigate the suitable system prediction horizon length, scenarios with various prediction horizon lengths were evaluated. It was found that, with inappropriate prediction lengths, the system performance will be poor because of either having insufficient time to implement countermeasures (prediction horizon too short) or being oversensitive to the variations of the traffic status change (prediction horizon too long). Among the tested values, 10 minutes appears to be the most stable and efficient length for the tested site. For improving the traffic in terms of both mobility and safety, a new objective function was proposed to consider both traffic mobility and safety costs during the system optimization. A real-time crash probability model was adopted to evaluate the safety risks. Different weighting parameters were tested to balance the priority between providing safety and mobility. Through the analysis of the evaluation results, a series of parameter values were suggested to be used for the DynaTAM system. Finally, after refining the system prediction horizon and adopting the new objective function with the safety consideration proposed in this study, the controlled system performance is compared with the uncontrolled case. It was observed that the traffic condition in the studied network improved significantly in terms of both traffic safety (overall crash probability was reduced by $39 \%$ ) and mobility (mobility cost reduced by $43 \%$ ).

\section{Conflict of Interests}

The author declares that there is no conflict of interests regarding the publication of this paper.

\section{References}

[1] Texas Transportation Institute, TTI's 2011 Urban Mobility Report Powered by INRIX Traffic Data, The Texas A\&M University System, 2011.

[2] American Automobile Association, "Crashes versus Congestion-What's the Cost to Society?” 2011.

[3] Transport Canada, "Further drop in road fatalities: 2007 Canadian motor vehicle traffic collision statistics," No. H036/10, 2010, http://www.tc.gc.ca/eng/motorvehiclesafety/tp-tp151451201.htm.

[4] Alberta Transportation, Alberta Traffic Collision Statistics 2008, Office of Traffic Safety, 2008, http://www.transportation.alberta .ca/Content/docType47/Production/2008AR.pdf.

[5] J. Fang, E. Hadiuzzaman, T. Qiu, M. Hadiuzzaman, and E. Yin, "DynaTAM: an online algorithm for performing simultaneously optimized proactive traffic control for freeways," Canadian Journal of Civil Engineering, vol. 41, no. 4, pp. 315-322, 2014.
[6] D. P. Masher, D. W. Ross, P. J. Wong, P. L. Tuan, P. L. Zeidler, and S. Peracek, "Guidelines for design and operating of ramp control systems," Stanford Research Institute Report NCHRP 3-22, SRI Project 3340, SRI, Menid Park, Calif, USA, 1975.

[7] M. Papageorgiou, H. Hadj-Salem, and F. Middelham, "ALINEA local ramp metering - summary of field results," Transportation Research Record, no. 1603, pp. 90-98, 1997.

[8] M. J. Cassidy and J. Rudjanakanoknad, "Increasing the capacity of an isolated merge by metering its on-ramp," Transportation Research Part B: Methodological, vol. 39, no. 10, pp. 896-913, 2005.

[9] M. Papageorgiou, I. Papamichail, A. D. Spiliopoulou, and A. F. Lentzakis, "Real-time merging traffic control with applications to toll plaza and work zone management," Transportation Research C: Emerging Technologies, vol. 16, no. 5, pp. 535-553, 2008.

[10] A. Kotsialos, M. Papageorgiou, M. Mangeas, and H. Haj-Salem, "Coordinated and integrated control of motorway networks via non-linear optimal control," Transportation Research C: Emerging Technologies, vol. 10, no. 1, pp. 65-84, 2002.

[11] A. Kotsialos and M. Papageorgiou, "Efficiency and equity properties of freeway network-wide ramp metering with AMOC," Transportation Research Part C, vol. 12, no. 6, pp. 401-420, 2004.

[12] I. Papamichail and M. Papageorgiou, "Traffic-responsive linked ramp-metering control," IEEE Transactions on Intelligent Transportation Systems, vol. 9, no. 1, pp. 111-121, 2008.

[13] I. Papamichail, M. Papageorgiou, V. Vong, and J. Gaffney, "Heuristic ramp-metering coordination strategy implemented at Monash Freeway, Australia," Transportation Research Record, vol. 2178, pp. 10-20, 2010.

[14] I. Papamichail, A. Kotsialos, I. Margonis, and M. Papageorgiou, "Coordinated ramp metering for freeway networks: a model-predictive hierarchical control approach," Transportation Research C, vol. 18, no. 3, pp. 311-331, 2010.

[15] H. Zackor, "Speed limitation on freeways: traffic-responsives trategies," in Concise Encyclopedia of Traffic and Transportation Systems, pp. 507-511, Pergamon Press, Oxford, UK, 1991.

[16] A. Hegyi, B. de Schutter, and J. Hellendoorn, "Optimal coordination of variable speed limits to suppress shock waves," IEEE Transactions on Intelligent Transportation Systems, vol. 6, no. 1, pp. 102-112, 2005.

[17] H. Wang, W. Wang, X. Chen, J. Chen, and J. Li, "Experimental features and characteristics of speed dispersion in urban freeway traffic," Transportation Research Record: Journal of the Transportation Research Board, vol. 1999, pp. 150-160, 2007.

[18] M. Abdel-Aty, N. Uddin, and A. Pande, "Split models for predicting multivehicle crashes during high-speed and low-speed operating conditions on freeways," Transportation Research Record, no. 1908, pp. 51-58, 2005.

[19] M. Abdel-Aty, J. Dilmore, and A. Dhindsa, "Evaluation of variable speed limits for real-time freeway safety improvement," Accident Analysis and Prevention, vol. 38, no. 2, pp. 335-345, 2006.

[20] M. A. Abdel-Aty and A. Dhindsa, "Coordinated use of variable speed limits and ramp metering for improving safety on congested freeways," in Proceedings of the 86th Annual Meeting of the Transportation Research Board, Washington, DC, USA.

[21] B. Hellinga and M. Mandelzys, "Impact of driver compliance on the safety and operational impacts of freeway variable speed limit systems," Journal of Transportation Engineering, vol. 137, no. 4, pp. 260-268, 2011. 
[22] J. Fang, A. Hadiuzzaman, Y. Luo, T. Qiu, and Md. Hadiuzzaman, "A novel VSL control strategy with traffic state prediction based collision probability assessments," Journal of the Transportation Research Board, 2014.

[23] A. Alessandri, A. di Febbraro, A. Ferrara, and E. Punta, "Optimal control of freeways via speed signalling and ramp metering," Control Engineering Practice, vol. 6, no. 6, pp. 771-780, 1998.

[24] A. Messmer and M. Papageorgiou, "METANET: a macroscopic simulation program for motorway networks," Traffic Engineering \& Control, vol. 31, no. 8-9, pp. 466-470, 1990.

[25] R. C. Carlson, I. Papamichail, M. Papageorgiou, and A. Messmer, "Optimal mainstream traffic flow control of large-scale motorway networks," Transportation Research C: Emerging Technologies, vol. 18, no. 2, pp. 193-212, 2010.

[26] X.-Y. Lu, P. Varaiya, R. Horowitz, D. Su, and S. E. Shladover, "Novel freeway traffic control with variable speed limit and coordinated ramp metering," Transportation Research Record, no. 2229, pp. 55-65, 2011.

[27] M. Tazul Islam, M. Hadiuzzaman, J. Fang, T. Qiu, and K. ElBasyouny, "Assessing mobility and safety impacts of a variable speed limit control strategy," Transportation Research Record, no. 2364, pp. 1-11, 2013. 


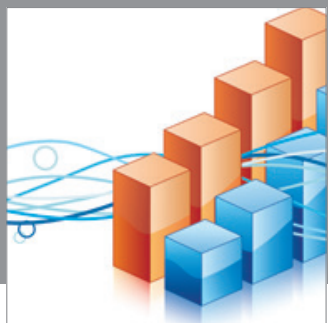

Advances in

Operations Research

mansans

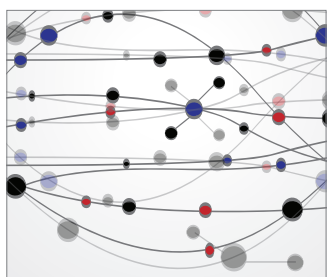

The Scientific World Journal
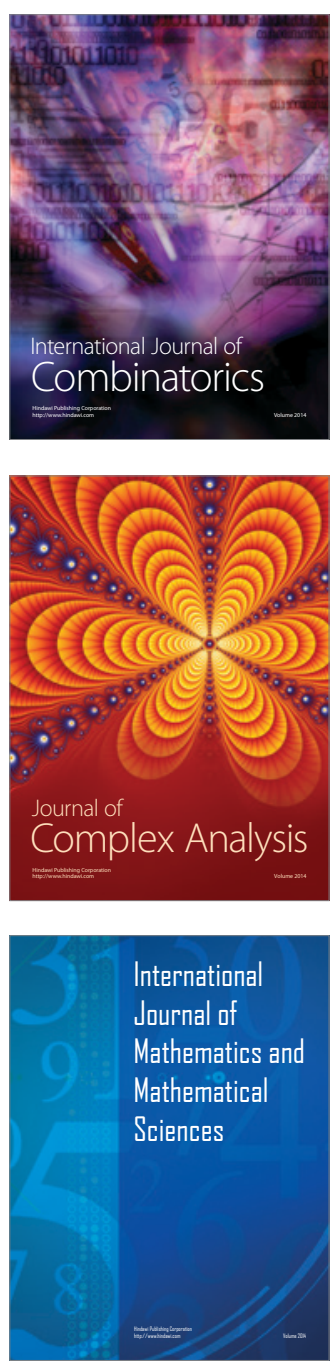
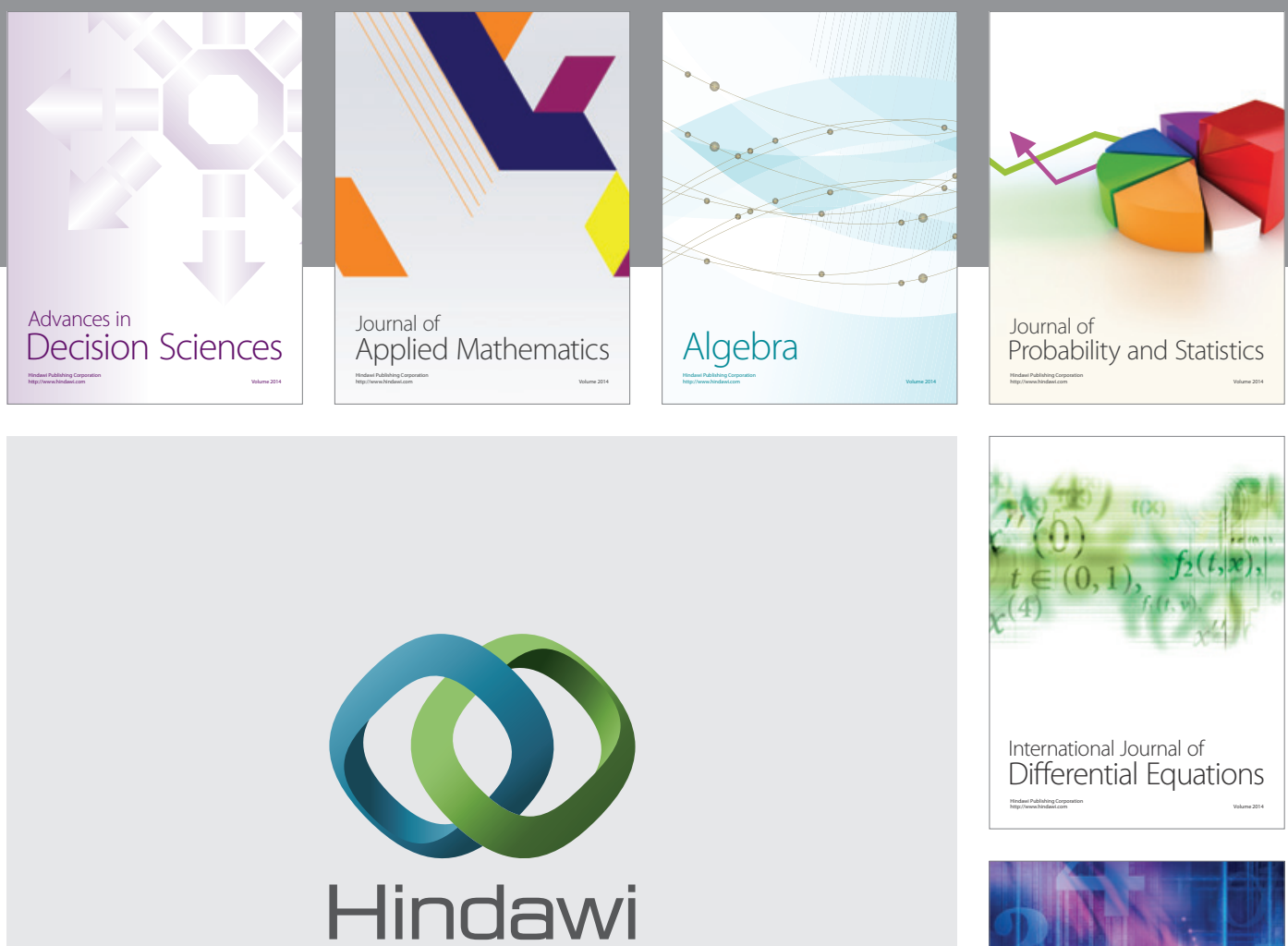

Submit your manuscripts at http://www.hindawi.com
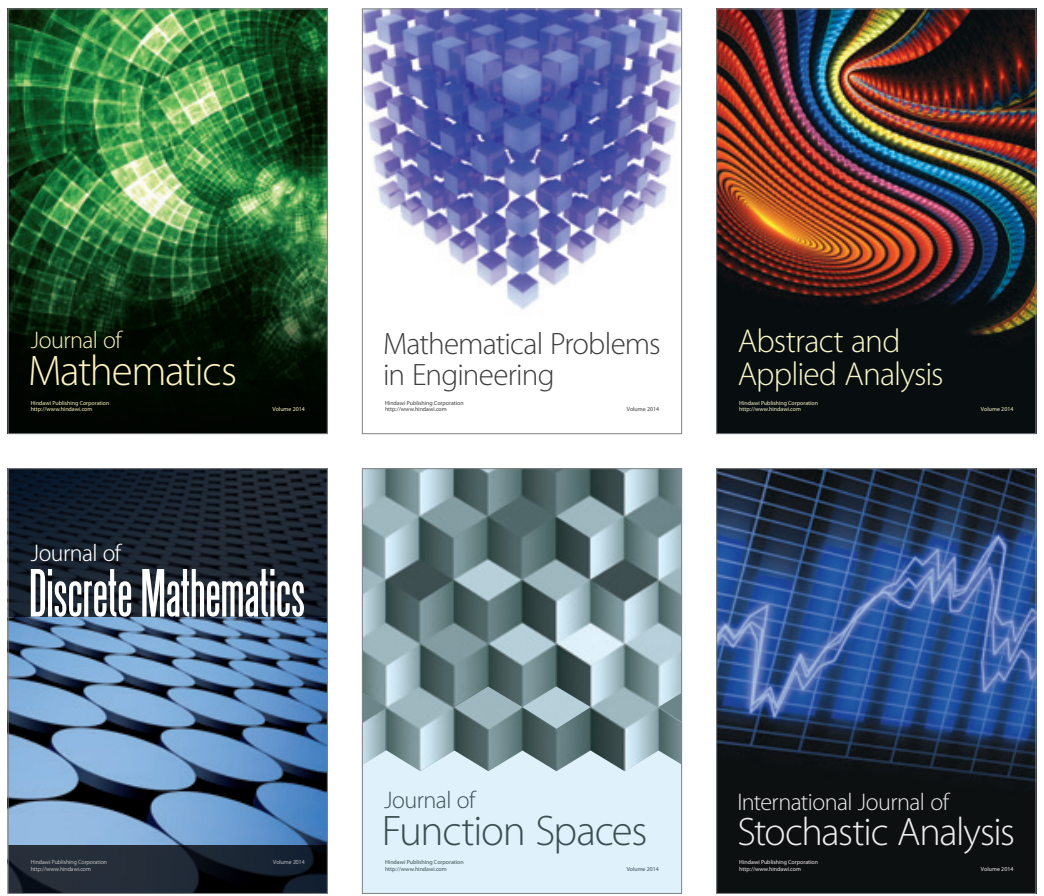

Journal of

Function Spaces

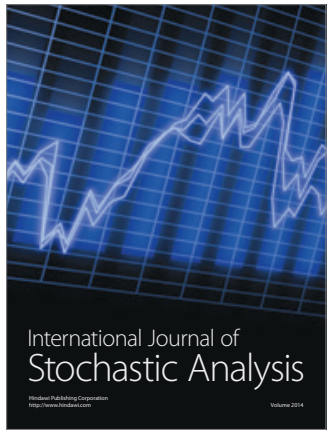

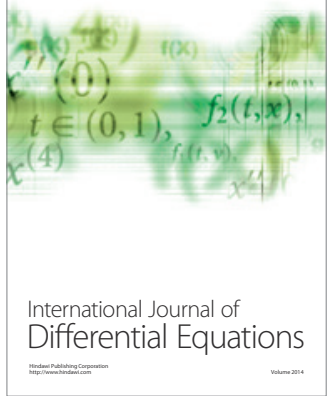
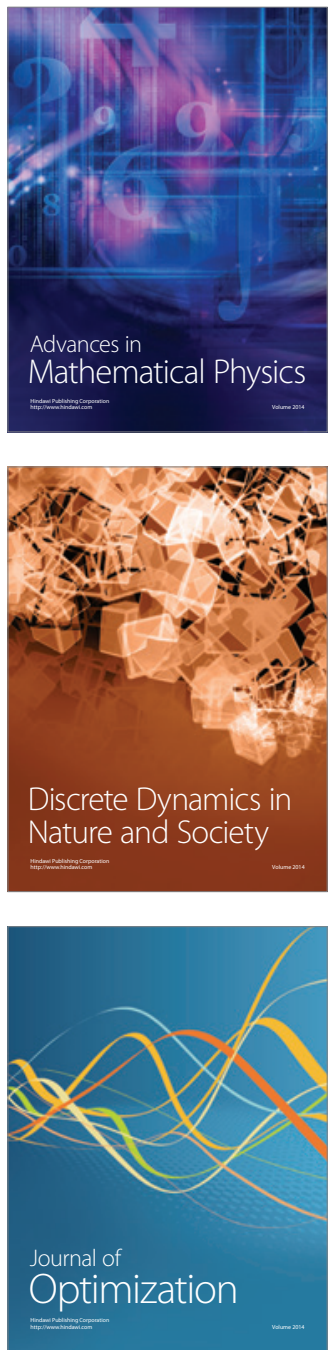